\title{
Raman Fingerprints of Atomically Precise Graphene Nanoribbons
}

\author{
Ivan A. Verzhbitskiy, ${ }^{\dagger}$ Marzio De Corato, ${ }^{\ddagger}$, Alice Ruini, ${ }^{\ddagger}, \S$ Elisa Molinari, ${ }^{\ddagger}, \S$ Akimitsu Narita, \\ Yunbin Hu,, Matthias G. Schwab, ${ }^{\|}$Matteo Bruna, ${ }^{\perp}$ Duhee Yoon, ${ }^{\perp}$ Silvia Milana, ${ }^{\perp}$ Xinliang Feng," \\ Klaus Müllen," Andrea C. Ferrari, ${ }^{\perp}$ Cinzia Casiraghi, ${ }^{*}, \dagger, \nabla$ and Deborah Prezzi ${ }^{*}, \S$
}

${ }^{\dagger}$ Physics Department, Free University of Berlin, 14195 Berlin, Germany

${ }^{\ddagger}$ Department of Physics, Mathematics, and Informatics, University of Modena and Reggio Emilia, 41121 Modena, Italy

${ }^{\S}$ Nanoscience Institute of CNR, S3 Center, 41125 Modena, Italy

"Max Planck Institute for Polymer Research, 55128 Mainz, Germany

${ }^{\perp}$ Cambridge Graphene Centre, University of Cambridge, Cambridge, CB3 OFA, United Kingdom

${ }^{\#}$ Center for Advancing Electronics Dresden (cfaed) and Department of Chemistry and Food Chemistry, Technische Universitaet Dresden, 01069 Dresden, Germany

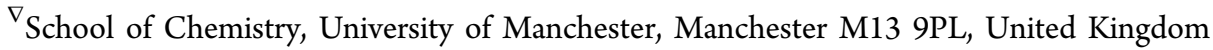

\section{Supporting Information}

ABSTRACT: Bottom-up approaches allow the production of ultranarrow and atomically precise graphene nanoribbons (GNRs) with electronic and optical properties controlled by the specific atomic structure. Combining Raman spectroscopy and ab initio simulations, we show that GNR width, edge geometry, and functional groups all influence their Raman spectra. The low-energy spectral region below $1000 \mathrm{~cm}^{-1}$ is particularly sensitive to edge morphology and functionalization, while the $\mathrm{D}$ peak dispersion can be used to uniquely fingerprint the presence of GNRs and differentiates them from other $\mathrm{sp}^{2}$ carbon nanostructures.
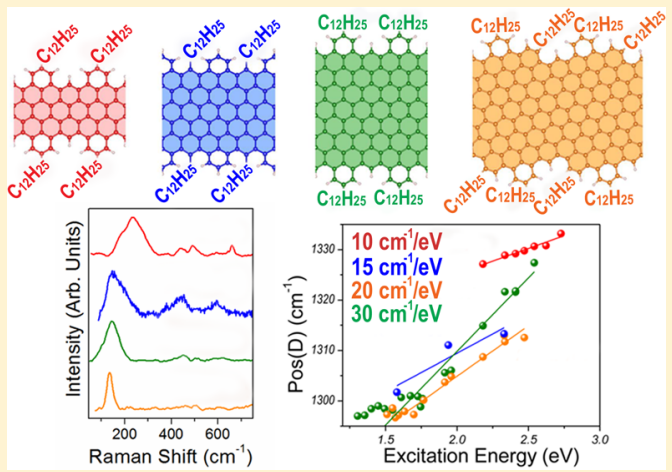

KEYWORDS: Graphene, nanoribbons, Raman spectroscopy

$\mathrm{R}$ aman spectroscopy is one of the most used characterization techniques in carbon science and technology. ${ }^{1-7}$ In the past decade, the rise of graphene has triggered extensive Raman studies to understand not only phonons but also electron-phonon, magneto-phonon, and electron-electron interactions, as well as the influence of the number and orientation of the layers in few-layer graphene (FLG), electric or magnetic fields, strain, doping, disorder, quality and type of edges, and functional groups. ${ }^{1}$ The Raman spectrum of singlelayer graphene (SLG) and FLG consists of two sets of peaks: the so-called $G$ and D peaks, which originate from in-plane vibrations and dominate the optical region of graphene and other $\mathrm{sp}^{2}$ bonded materials, ${ }^{8,9}$ and the low-energy peaks resulting from the relative motion of the planes, such as the shear $^{10}$ (C) and the layer-breathing modes ${ }^{11-13}$ (LBMs), which can be used as a direct probe of the number of layers.

The low-energy region where the C and LBMs are located is particularly interesting because specific fingerprints have been observed for a variety of carbon allotropes. Most notably, in the case of carbon nanotubes (CNTs) a characteristic peak at low energy, associated with the radial breathing mode (RBM) of all the atoms of the structure, has been widely exploited to determine the tube diameter. ${ }^{14}$ Polycyclic aromatic hydrocarbons (PAHs) are also characterized by breathing-like lowenergy modes that can be related to their lateral size. ${ }^{15,16}$ GNRs, which are of interest for emerging digital nanoelectronics, optoelectronics, and spintronics, ${ }^{17-19}$ are also expected to show characteristic Raman features in this spectral region due to their finite width and low symmetry. ${ }^{20-25}$

Recently, techniques based on surface-assisted ${ }^{26}$ or solutionbased $^{27-29}$ cyclo-dehydrogenation of tailor-made aromatic polymer precursors have enabled the production of welldefined GNRs with lateral width well below $10 \mathrm{~nm}$ and defined edge patterns. ${ }^{26-31}$ In the case of GNRs prepared by solutionbased processing, the edges are typically functionalized by alkyl chains to improve solubility. ${ }^{29}$ Thus, these GNRs provide a unique opportunity to study the combined effect of finite width and edge patterns and gain insight into the evolution of the vibrational properties with lateral size. At the same time, such GNRs call for the identification of specific Raman fingerprints,

Received: October 14, 2015

Revised: February 16, 2016

Published: February 23, 2016 


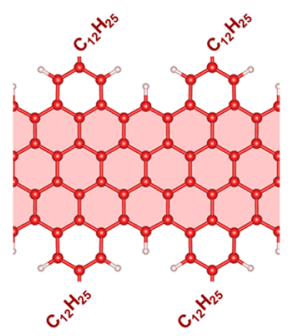

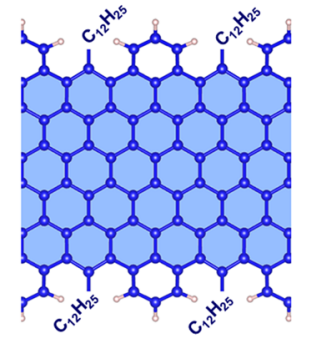

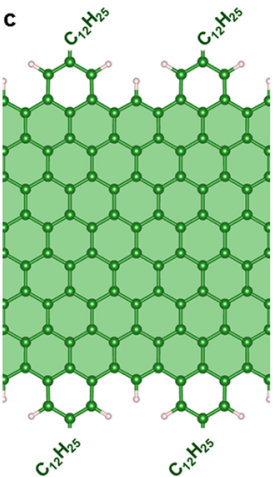

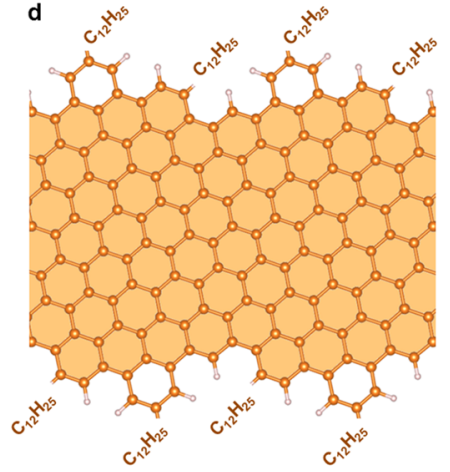

Figure 1. Structural model of the GNRs. (a-d) Ball-and-stick representation of the atomic structure of the cove-shaped GNRs investigate here, that is, 4CNR (a), 6CNR (b), $8 \mathrm{CNR}$ (c), and $m$-ANR (d). The schematics show the characteristic variable width of these GNRs and the location of the alkyl side chains in each case. The shaded areas indicate the corresponding zigzag/chiral GNRs.
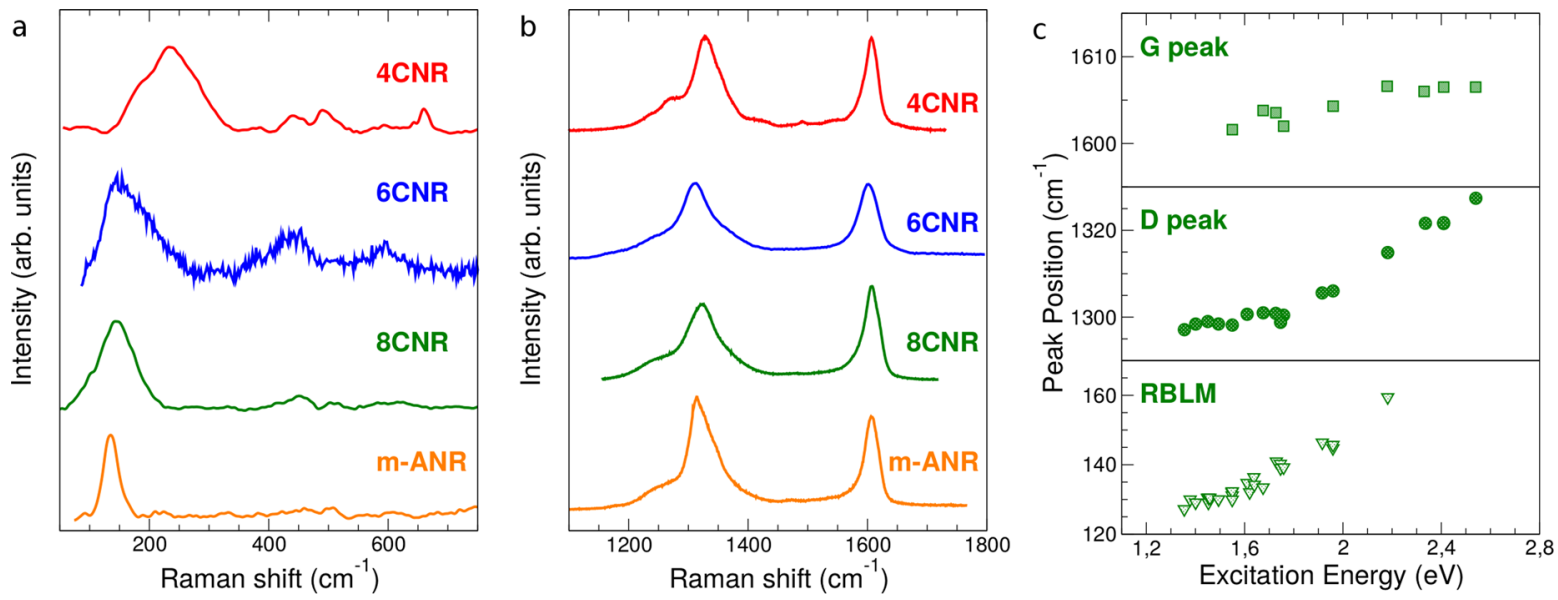

Figure 2. Raman spectra of cove-shaped GNRs. (a) Acoustic and (b) optical region of the Raman spectrum for the cove-shaped GNRs in Figure 1. The 4CNR and 6CNR were excited at $\sim 2.4 \mathrm{eV}$, while $8 \mathrm{CNR}$ and $m$-ANR were excited at $\sim 1.9 \mathrm{eV}$. (c) Peak dispersion of $8 \mathrm{CNR}$ as a function of excitation energy for the G (top) and D peaks (middle), as well as for the RLBM (bottom).

which could be extremely relevant for their characterization and design, similar to what happened in the case of CNTs.

In this respect, experiments on GNRs mostly targeted peaks in the $\mathrm{D} / \mathrm{G}$ energy region. ${ }^{27,31-33}$ However, these features are usually just discussed as a hallmark of $\mathrm{sp}^{2}$ hybridization, and no further analysis exists so far. A few groups studied the lowenergy GNR Raman bands. Cai et al. ${ }^{26}$ reported a sharp (20$30 \mathrm{~cm}^{-1}$ ) low-energy peak at $\sim 400 \mathrm{~cm}^{-1}$, and assigned it to a radial-like breathing mode ${ }^{20}$ (RLBM), where all the atoms of the ribbon move in-plane along the ribbon width direction in analogy to the RBM in CNTs. Because of that, the position of this peak is determined by the GNR width only. ${ }^{20-25} \mathrm{~A}$ similar feature was reported in refs 29 and 30 for other GNRs produced by solution-based processing but much broader ( $\sim 100 \mathrm{~cm}^{-1}$ in width) when compared to ref 26 , with several subcomponents, and downshifted $\sim 50 \mathrm{~cm}^{-1}$ when compared to what was expected from the inverse dependence of the RLBM frequency with GNR width. ${ }^{20-25}$ This may indicate a more complex dependence of the RLBM on GNR width and edge type and call for further investigations.

Here, we report a combined experimental and theoretical study of the Raman spectrum of ultranarrow $(<2 \mathrm{~nm})$ GNRs with well-defined edges, produced by solution-based methods. By investigating four GNRs with different width, edge-pattern, and location of the alkyl groups, we demonstrate that the RLBM can be influenced by the edge termination and not just by the width. We also show that the $\mathrm{D}$ peak dispersion with excitation energy is a further fingerprint of these GNRs and uniquely differentiates them from other $\mathrm{sp}^{2}$ carbon systems such as graphene or CNTs.

We investigate four GNRs, which are functionalized with long alkyl chains $\left(-\mathrm{C}_{12} \mathrm{H}_{25}\right)$ to favor their solubility. ${ }^{30}$ The structures in Figure $1 \mathrm{a}-\mathrm{c}$ represent a series of "cove-shaped" GNRs with the same edge pattern and increasing width, where a benzo ring periodically decorates the zigzag (ZZ) edge. Following ref 34 , these structures are labeled $n$ CNRs, where $n$ indicates the width of the ZZ GNR core (here $n=4,6,8$ ). In addition to those samples, we also investigate a GNR based on the chiral-edged $(4,1)$-GNR with periodically fused benzo rings. This is called m-ANR in ref 34 and shown here in Figure 1d. The $m$-ANR has the same width as the $8 \mathrm{CNR}$, but its "chirality" leads to slightly different edge pattern. The 6CNR has intermediate width between $4 \mathrm{CNR}$ and $8 \mathrm{CNR}$ and the same edge pattern, but the alkyl groups are placed in a different location at edge, i.e. 4- and 8CNR are substituted at the outer positions on the benzo rings, whereas the $6 \mathrm{CNR}$ is substituted at the inner position inside the cove-type edge.

Low-Energy Spectral Region and Radial-Like Breathing Mode. Figure 2a shows the low-energy Raman spectrum of the GNRs in Figure 1. In the case of 4CNR, one broad peak appears at $\sim 230 \mathrm{~cm}^{-1}$ (full width at half-maximum, fwhm $\sim 100$ $\mathrm{cm}^{-1}$ ), which comprises at least three contributions. For 6CNR, 

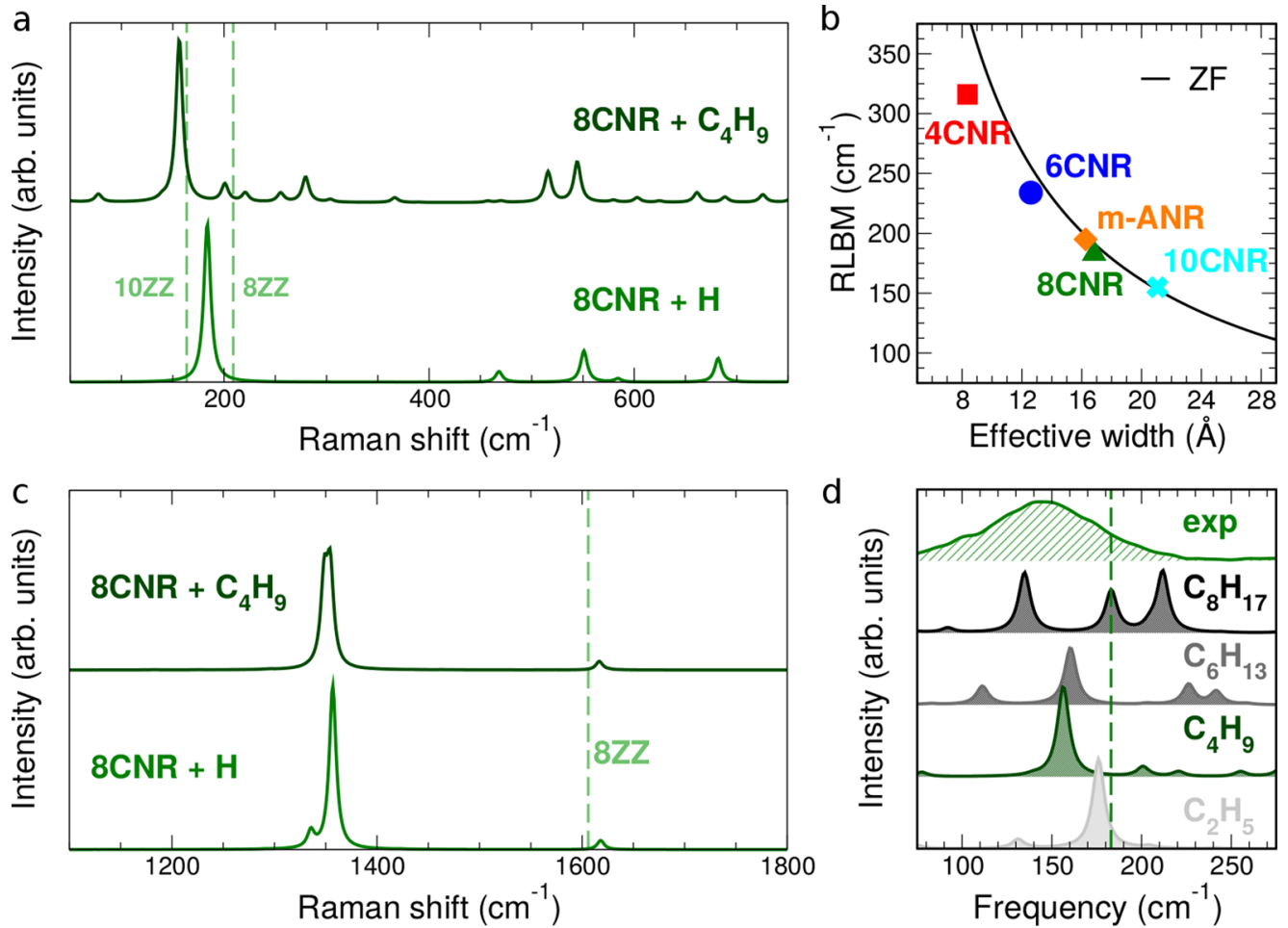

Figure 3. Simulated vibrational properties of cove-shaped GNRs. (a) Acoustic and (c) optical region of the Raman spectrum of $8 \mathrm{CNR}$, as resulting from ab initio DFPT simulations. The spectrum is shown for both hydrogen-terminated $(+\mathrm{H}$, green $)$ and functionalized $8 \mathrm{CNR}\left(+\mathrm{C}_{4} \mathrm{H}_{9}\right.$, dark green). The dashed lines indicate the position of the RLBM for 8- and 10-ZGNRs (labeled 8-ZZ and 10-ZZ, respectively, light green) and the position of the G peak for 8-ZGNR. (b) The frequency of the RLBM calculated from first principles for several H-passivated cove-shaped GNRs is compared to the result of the ZF approximation (black curve) as a function of the GNR effective width. (d) The low-energy spectral region of the functionalized $8 \mathrm{CNR}$ is shown as a function of the chain length and compared with experimental data. The dashed line indicates the frequency of the RLBM for the $\mathrm{H}$-terminated system. For convenience, a small Lorentzian broadening of $\sim 10 \mathrm{~cm}^{-1}$ is introduced in all spectra.

$8 \mathrm{CNR}$, and $m$-ANR, the most intense peak is located at $\sim 130-$ $150 \mathrm{~cm}^{-1}$ and its width is sharp for $m$-ANR $\left(\sim 30 \mathrm{~cm}^{-1}\right)$, whereas it is larger for $8 \mathrm{CNR}$ and 6CNR $\left(\sim 80-100 \mathrm{~cm}^{-1}\right)$. Furthermore, depending on the exact GNR structure, the peak disappears at certain excitation energies. For instance, the peak is not seen at energies below $1.96 \mathrm{eV}$ for $4 \mathrm{CNR}$, while for $8 \mathrm{CNR}$ it is detected at $1.96 \mathrm{eV}$. Other peaks with small intensity are also seen in the spectral range $400-700 \mathrm{~cm}^{-1}$, as shown in Figure 2a. This is different from the case of CNTs and armchair GNRs grown on substrate, ${ }^{26}$ where the low energy region is dominated by a sharp breathing mode with frequency uniquely determined by the CNT/GNR lateral size and described by the zone-folding (ZF) approximation. ${ }^{24,25}$

In order to gain further insights into the origin of the GNRs Raman peaks at low energy, we have computed from first principles the Raman spectra of several GNRs (see Methods) by increasing the structure complexity in a stepwise manner. We first consider perfect ZZ-GNRs, corresponding to the cores of the cove-shaped ribbons studied here (indicated by the shaded areas in Figure $1 \mathrm{a}-\mathrm{c}$ ). We then study cove-shaped GNRs with hydrogen-terminated edges. Finally, we compare the above systems with functionalized ones, as in the real samples, though simulated by using shorter alkyl chains $\left(-\mathrm{C}_{4} \mathrm{H}_{9}\right.$ instead of $\left.-\mathrm{C}_{12} \mathrm{H}_{25}\right)$ to make the calculations more affordable. Figure $3 \mathrm{a}$ shows the simulated Raman spectrum of the $8 \mathrm{CNR}$, while the spectra of the other GNRs are reported in Supporting Information. Let us first focus on the H-passivated $8 \mathrm{CNR}$, hereafter labeled $8 \mathrm{CNR}+\mathrm{H}$. The spectrum is characterized by a dominant peak at $183 \mathrm{~cm}^{-1}$, which falls in-between the RLBMs of the ZZ-GNRs corresponding to the minimum and maximum width of this cove-shaped GNR, that is, 209 and $164 \mathrm{~cm}^{-1}$ for the 8- and 10-ZZ GNRs, respectively. This behavior, common to all the GNRs studied here, can be understood in terms of an effective width model. Indeed, these cove-shaped GNRs present a modulated structure with variable width (e.g., 4CNR core width ranges from 0.69 to $1.13 \mathrm{~nm}$ ). However, we can define an effective width as the weighted average of the different GNR widths (see Supporting Information) and compare our first principles simulations with the results obtained by ZF. Figure $3 \mathrm{~b}$ shows that the agreement is within less than $10 \mathrm{~cm}^{-1}$ for GNRs wider than 15 $\AA$, as expected for this approximation. ${ }^{24,25}$ Besides the RLBM, there are higher frequency modes $\left(470-680 \mathrm{~cm}^{-1}\right)$, combining longitudinal and transverse components. These modes, not present in ZZ-GNRs, appear in cove-shaped GNRs in view of the different periodicity along the ribbon axis introduced by the additional benzo rings at the edge.

We next functionalize the edges with short alkyl chains $\left(8 \mathrm{CNR}+\mathrm{C}_{4} \mathrm{H}_{9}\right)$. We observe a further red-shift of the RLBM peak, which moves from 183 to $156 \mathrm{~cm}^{-1}$, close to that of the

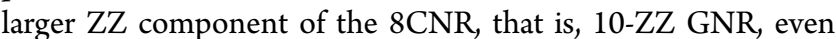
though the origin of the shift is rather different, as clarified by our analysis. The breathing does not involve the ribbon only but also part of the chain that moves in phase with the ribbon atoms. This causes a redshift as a result of an increase in the effective width. We further check the effect of the chains by varying the chain length, as shown in Figure $3 \mathrm{~d}$. In addition to the effect explained above with a redshift that depends on the 
chain length, we find that, depending on both the chain length and the GNR width, the coupling with the chain modes can give rise to different subpeaks (see, e.g., the case of $\mathrm{C}_{8} \mathrm{H}_{17}$, Figure $3 \mathrm{~d}$ ). The relaxation of the system symmetry allows for both a mixing of longitudinal $(\mathrm{L})$, transverse $(\mathrm{T})$, and normal (Z) modes and the activation of modes otherwise forbidden. All of this can explain the broadening of the low-frequency peak observed experimentally, which is indeed different for GNRs of different width.

High-Energy Spectral Region: D and G Peaks, Overtones, and Combination Modes. Figure $2 \mathrm{~b}$ shows the first order Raman spectrum of the ribbons measured at $2.41 \mathrm{eV}$ with $\mathrm{D}$ and $\mathrm{G}$ peaks typical of $\mathrm{C} \mathrm{sp}^{2}$ materials. ${ }^{1-6}$ However, the $\mathrm{G}$ peak, which corresponds to the high frequency $E_{2 g}$ phonon at $\Gamma$, is up-shifted $\left(\sim 1605 \mathrm{~cm}^{-1}\right)$ and broader if compared to pristine graphene $\left(\mathrm{fwhm} \sim 25 \mathrm{~cm}^{-1}\right.$ ). Similar results were observed in small graphite domains ${ }^{35}$ and $\mathrm{PAH}^{6}$ due to the relaxation of the momentum conservation induced by finite size. Moreover, we do not observe any splitting of the $\mathrm{G}$ peak, as usually found instead for CNTs, ${ }^{4,5}$ where this mode, which is doubly degenerate in graphene, ${ }^{36}$ splits into a longitudinaloptical (LO) and a transverse-optical (TO) component. Our first principles simulations show that the $G$ peak, located at $1618 \mathrm{~cm}^{-1}$ for the $8 \mathrm{CNR}$ (Figure 3c), is mainly due to the TO mode with smaller contributions due to TO overtones (within $10 \mathrm{~cm}^{-1}$ ); the LO mode is instead inactive in backscattering, as expected in purely ZZ-GNRs. ${ }^{24}$ The presence of the side chains does not alter significantly the nature and position of the main features in the high-energy region of the spectrum, mostly dictated by the edge morphology, as seen by comparing the curves in Figure 3c. Note however that a different position of the functional chain along the GNR edge, as in the case of 6CNR, can induce a distortion of the GNR backbone, thus influencing the peak position (see simulated spectra of 6CNR in Supporting Information).

Figure $2 \mathrm{~b}$ also shows a prominent $\mathrm{D}$ peak that is characterized by a dominant component at about 1310-1330 $\mathrm{cm}^{-1}$ with an intensity comparable to that of the $\mathrm{G}$ peak and by one or more shoulders at lower wavenumbers. The Raman shifts of these features are in agreement with our first principles simulations, which show a structured peak in this region with two main components (at 1336 and $1357 \mathrm{~cm}^{-1}$ for the $8 \mathrm{CNR}$, Figure $3 \mathrm{c}$ ) corresponding to the breathing modes of six-atom rings. This behavior makes the spectrum resemble that of defective graphene. ${ }^{37-41}$ However, there are notable differences that allow us to clearly distinguish our GNRs from defective graphene. The most striking one is found by analyzing the energy dependence of the $\mathrm{D}$ peak. In graphene, the $\mathrm{D}$ peak, coming from $\mathrm{TO}$ phonons around the Brillouin Zone (BZ) edge $K$, is activated by an intervalley double resonance process $^{42}$ in the presence of defects, and it is strongly dispersive with excitation energy due to a Kohn Anomaly at $\mathrm{K}^{43}$ The typical $\mathrm{D}$ peak dispersion of graphene is $\sim 50 \mathrm{~cm}^{-1} / \mathrm{eV}^{44,45} \mathrm{In}$ the case of GNRs, we measure different D-peak dispersions for the different GNRs (see Supporting Information). In particular, due to its low band gap $\left(\sim 1.2 \mathrm{eV}^{30}\right)$ the $8 \mathrm{CNR}$ allows performing Raman spectroscopy in a wide range of energies (from 1.3 to $2.54 \mathrm{eV}$ ) without any photoluminescence background hiding the Raman peaks (Figure 2c). If we fit the dispersion with two slopes, we get about 7 and $35 \mathrm{~cm}^{-1} / \mathrm{eV}$ for the low $(<1.8 \mathrm{eV})$ and high $(>1.8 \mathrm{eV})$ excitation energy region, respectively. A considerable dispersion is found also for the RLBM (Figure 2c, bottom panel), while the G peak shows a very small dispersion at the limit of the spectrometer resolution (Figure 2c, top panel). In addition to this, we observe the $\mathrm{G}+\mathrm{D}$ combination mode and the $2 \mathrm{G}$ mode, which are not observed in defective graphene. A systematic comparison between defective graphene and our GNRs is reported in the Supporting Information.

To fully understand these experimental observations, one needs to consider that several factors come into play when we move from graphene to narrow GNRs. First, we have neither Kohn anomalies (see calculated phonon dispersion in Supporting Information) nor linear electronic dispersion in such GNRs, which have semiconducting character, with an optical response dominated by excitonic effects, ${ }^{46,47}$ especially for excitation energies close to the optical gap. Second, the $\mathrm{K}$ point folds onto $\Gamma$ in these cove-shaped GNRs; ${ }^{34}$ as such, we do not need a double-resonance process to activate this mode but just a first-order process like in armchair GNRs, where the $\mathrm{D}$ peak is also observed. ${ }^{26}$ For the same reason, we are also able to observe the $\mathrm{G}+\mathrm{D}$ mode. In addition, one would expect a nondispersive behavior for the $D$ peak, similar to the $G$ peak in graphene. The observed dispersion is thus related to some disorder-induced scattering, for example, due to edge functionalization, defects formed during the GNR production, or length distribution.

In conclusion, we combined an experimental and theoretical analysis of the Raman spectra of ultranarrow and structurally well-defined graphene nanoribbons with cove-type edges. The low-frequency spectral region contains the main fingerprints of these materials. By analyzing the differences with respect to other systems, such as an ideal ZZGNR and the same GNR without lateral chains ( $\mathrm{H}$ passivated), ab initio simulations show that the number of Raman peaks and their position are crucially affected by edge modifications. The full description of cove-type edge and alkyl chains is fundamental to get an agreement with experiments because both contribute to the shift and splitting of the peaks as well as to a redistribution of the Raman intensity. The RLBM is especially sensitive, not simply to the width, but also to the edge modulation and functionalization, making it very different from the ideal cases studied to date, where the RLBM does not show significant dependence on edge type. The high-energy spectral region appears similar to that of defective graphene with D and G peaks of comparable intensity. However, the presence of the $D$ $+\mathrm{G}$ combination mode and the different D-peak dispersion allow us to clearly distinguish these GNRs from defected graphene and other graphitic materials.

Methods. Experimental Details. Details on the preparation of $4 \mathrm{CNR}, 8 \mathrm{CNR}$, and $m$-ANR are described in refs $28-30$. The synthesis of 6CNR will be reported elsewhere. Raman measurements are performed with a combination of different spectrometers (Witec confocal spectrometer, Renishaw InVia, and TK64000 by Horiba). A 100X objective is used and the power on the sample is below $0.1 \mathrm{~mW}$ to avoid damage. The Raman spectra have been measured with at least three accumulations and in at least three different points on the same sample. Measurements were repeated with different spectrometers in different laboratories in different countries to avoid any experimental artifact. The samples are measured as powder in resonance condition [the optical gap is $\sim 1.9, \sim 1.2$, and $\sim 1.12 \mathrm{eV}$ for 4-CNR, 8-CNR, and m-ANR, respectively, ${ }^{28-30}$ whereas for $6 \mathrm{CNR}$ it is $\left.\sim 1.8 \mathrm{eV}\right]$.

Computational Approach. Simulations are performed using a first principles plane-wave pseudopotential implementation of 
density functional theory and density functional perturbation theory (DFPT), ${ }^{48}$ as available in the Quantum ESPRESSO package. ${ }^{49}$ The local density approximation for the exchangecorrelation functional is used. Raman intensities are calculated within the Placzek approximation (non-resonant condition) by using the second-order response method in ref 50. This approach is known to give accurate Raman shifts, whereas the relative intensity of the peaks cannot be directly compared with resonance Raman experiments. Norm-conserving pseudopotentials are employed with a plane-wave cutoff energy of $70 \mathrm{Ry}$. A vacuum region of $12 \AA$ in the nonperiodic directions is introduced to prevent interaction between periodic images. The atomic positions are fully relaxed until forces are less than $5 \times$ $10^{-4}$ au. Phonon frequencies and Raman tensor are calculated using a $16 \times 1 \times 1 k$-point grid.

\section{ASSOCIATED CONTENT}

\section{S Supporting Information}

The Supporting Information is available free of charge on the ACS Publications website at DOI: 10.1021/acs.nanolett.5b04183.

Detailed descriptions of (I) the effect of the laser power on the first order Raman spectrum of the $4 \mathrm{CNR}+\mathrm{C}_{12} \mathrm{H}_{25}$ ribbon; (II) multiwavelength Raman spectroscopy of GNRs as compared to defective graphene; (III) the Raman spectra (experimental and simulated) of all ribbons with different side chains and chain locations; (IV) the zone-folding approximation for cove-shaped GNRs; (V) the D- and G-peak dispersions from DFPT. (PDF)

\section{AUTHOR INFORMATION}

\section{Corresponding Authors}

*E-mail: (D.P.) deborah.prezzi@nano.cnr.it.

*E-mail: (C.C.) cinzia.casiraghi@manchester.ac.uk.

\section{Present Addresses}

(I.A.V.) Physics Department, National University of Singapore, 117542, Singapore.

(M.G.S.) BASF SE, Carl-Bosch-Straße 38, 67056 Ludwigshafen, Germany.

\section{Author Contributions}

I.A.V., M.B., D.Y., and S.M. performed the Raman measurements with A.C.F. and C.C. providing supervision. M.D.C. A.R., E.M., and D.P. provided the theoretical framework. A.N., Y.H., and M.G.S. prepared the graphene nanoribbons under the supervision of X.F and K.M. C.C, D.P., and A.C.F wrote the manuscript with inputs from all authors.

\section{Notes}

The authors declare no competing financial interest.

\section{ACKNOWLEDGMENTS}

We acknowledge funding from the Alexander von Humboldt Foundation in the framework of the Sofja Kovalevskaja Award, endowed by the Federal Ministry for Education and Research of Germany; the ESF project GOSPEL (ref. no. 09-EuroGRAPHENE-FP-001); the European Research Council (Grant NOC-2D, NANOGRAPH, and Hetero2D); the Italian Ministry of Research through the national projects PRINGRAF (Grant 20105ZZTSE) and FIRB-FLASHit (Grant RBFR12SWOJ); the DFG Priority Program SPP 1459; the Graphene Flagship (ref. no. CNECT-ICT-604391); the EU project MoQuaS; EPSRC Grants (EP/K01711X/1, EP/ K017144/1); the EU grant GENIUS; a Royal Society Wolfson Research Merit Award. Computer time was granted by PRACE at the CINECA Supercomputing Center (Grant PRA06 1348), and by the Center for Functional Nanomaterials at Brookhaven National Laboratory, supported by the U.S. Department of Energy, Office of Basic Energy Sciences, under contract number DE-SC0012704.

\section{REFERENCES}

(1) Ferrari, A. C.; Basko, D. M. Nat. Nanotechnol. 2013, 8 (4), 235246.

(2) Casiraghi, C. In Spectroscopic Properties of Inorganic and Organometallic Compounds: Techniques, Materials and Applications; The Royal Society of Chemistry: London, 2012; Vol. 43, pp 29-56.

(3) Jorio, A.; Saito, R.; Dresselhaus, G.; Dresselhaus, M. S. Raman Spectroscopy in Graphene Related Systems; Wiley-VCH Verlag GmbH: Berlin, 2011.

(4) Saito, R.; Dresselhaus, G.; Dresselhaus, M. S. Physical Properties of Carbon Nanotubes; Imperial College Press: London, 1998.

(5) Reich, S.; Thomsen, C.; Maultzsch, J. Carbon Nanotubes: Basic Concepts and Physical Properties; Wiley-VCH Verlag GmbH: Berlin, 2004.

(6) Castiglioni, C.; Tommasini, M.; Zerbi, G. Philos. Trans. R. Soc., A 2004, 362 (1824), 2425-2459.

(7) Ferrari, A. C.; Robertson, J. Philos. Trans. R. Soc., A 2004, 362 (1824), 2477-2512.

(8) Tuinstra, F. J. Chem. Phys. 1970, 53 (3), 1126.

(9) Ferrari, A. C. Solid State Commun. 2007, 143 (1-2), 47-57.

(10) Tan, P. H.; Han, W. P.; Zhao, W. J.; Wu, Z. H.; Chang, K.; Wang, H.; Wang, Y. F.; Bonini, N.; Marzari, N.; Pugno, N.; Savini, G.; Lombardo, A.; Ferrari, A. C. Nat. Mater. 2012, 11 (4), 294-300.

(11) Lui, C. H.; Malard, L. M.; Kim, S.; Lantz, G.; Laverge, F. E.; Saito, R.; Heinz, T. F. Nano Lett. 2012, 12 (11), 5539-5544.

(12) Wu, J.-B.; Zhang, X.; Ijäs, M.; Han, W.-P.; Qiao, X.-F.; Li, X.-L.; Jiang, D.-S.; Ferrari, A. C.; Tan, P.-H. Nat. Commun. 2014, 5, 5309.

(13) Wu, J.-B.; Hu, Z.-X.; Zhang, X.; Han, W.-P.; Lu, Y.; Shi, W.; Qiao, X.-F.; Ijiäs, M.; Milana, S.; Ji, W.; Ferrari, A. C.; Tan, P.-H. ACS Nano 2015, 9 (7), 7440-7449.

(14) Rao, A. M.; Richter, E.; Bandow, S.; Chase, B.; Eklund, P. C.; Williams, K. A.; Fang, S.; Subbaswamy, K. R.; Menon, M.; Thess, A.; Smalley, R. E.; Dresselhaus, G.; Dresselhaus, M. S. Science 1997, 275 (5297), 187-191.

(15) Maghsoumi, A.; Brambilla, L.; Castiglioni, C.; Müllen, K.; Tommasini, M. J. Raman Spectrosc. 2015, 46 (9), 757-764.

(16) Di Donato, E.; Tommasini, M.; Fustella, G.; Brambilla, L.; Castiglioni, C.; Zerbi, G.; Simpson, C. D.; Müllen, K.; Negri, F. Chem. Phys. 2004, 301 (1), 81-93.

(17) Terrones, M.; Botello-Méndez, A. R.; Campos-Delgado, J.; López-Urías, F.; Vega-Cantú, Y. I.; Rodríguez-Macías, F. J.; Elías, A. L.; Muñoz-Sandoval, E.; Cano-Márquez, A. G.; Charlier, J.-C. Nano Today 2010, 5 (4), 351-372.

(18) Yazyev, O. V. Acc. Chem. Res. 2013, 46 (10), 2319-2328.

(19) Ferrari, A. C.; Bonaccorso, F.; Fal'ko, V.; Novoselov, K. S.; Roche, S.; Bøggild, P.; Borini, S.; Koppens, F. H. L.; Palermo, V.; Pugno, N.; Garrido, J. A.; Sordan, R.; Bianco, A.; Ballerini, L.; Prato, M.; Lidorikis, E.; Kivioja, J.; Marinelli, C.; Ryhänen, T.; Morpurgo, A.; et al. Nanoscale 2015, 7 (11), 4598-4810.

(20) Zhou, J.; Dong, J. Appl. Phys. Lett. 2007, 91 (17), 173108.

(21) Yamada, M.; Yamakita, Y.; Ohno, K. Phys. Rev. B: Condens. Matter Mater. Phys. 2008, 77 (5), 54302.

(22) Vandescuren, M.; Hermet, P.; Meunier, V.; Henrard, L.; Lambin, P. Phys. Rev. B: Condens. Matter Mater. Phys. 2008, 78 (19), 195401.

(23) Gillen, R.; Mohr, M.; Thomsen, C.; Maultzsch, J. Phys. Rev. B: Condens. Matter Mater. Phys. 2009, 80 (15), 155418.

(24) Gillen, R.; Mohr, M.; Maultzsch, J. Phys. Status Solidi B 2010, 247 (11-12), 2941-2944. 
(25) Gillen, R.; Mohr, M.; Maultzsch, J. Phys. Rev. B: Condens. Matter Mater. Phys. 2010, 81 (20), 205426.

(26) Cai, J.; Ruffieux, P.; Jaafar, R.; Bieri, M.; Braun, T.; Blankenburg, S.; Muoth Matthiasand Seitsonen, A. P.; Saleh, M.; Feng, X.; Muellen, K.; Fasel, R. Nature (London, U. K.) 2010, 466, 470-473.

(27) Dössel, L.; Gherghel, L.; Feng, X.; Muellen, K. Angew. Chem., Int. Ed. 2011, 50 (11), 2540-2543.

(28) Schwab, M. G.; Narita, A.; Hernandez, Y.; Balandina, T.; Mali, K. S.; De Feyter, S.; Feng, X.; Müllen, K. J. Am. Chem. Soc. 2012, 134 (44), 18169-18172.

(29) Narita, A.; Feng, X.; Hernandez, Y.; Jensen, S. A.; Bonn, M.; Yang, H.; Verzhbitskiy, I. A.; Casiraghi, C.; Hansen, M. R.; Koch, A. H. R.; Fytas, G.; Ivasenko, O.; Li, B.; Mali, K. S.; Balandina, T.; Mahesh, S.; De Feyter, S.; Müllen, K. Nat. Chem. 2013, 6 (2), 126-132.

(30) Narita, A.; Verzhbitskiy, I. A.; Frederickx, W.; Mali, K. S.; Jensen, S. A.; Hansen, M. R.; Bonn, M.; De Feyter, S.; Casiraghi, C.; Feng, X.; Müllen, K. ACS Nano 2014, 8 (11), 11622-11630.

(31) Schwab, M. G.; Narita, A.; Osella, S.; Hu, Y.; Maghsoumi, A.; Mavrinsky, A.; Pisula, W.; Castiglioni, C.; Tommasini, M.; Beljonne, D.; Feng, X.; Müllen, K. Chem. - Asian J. 2015, 10 (10), 2134-2138.

(32) Abbas, A. N.; Liu, G.; Narita, A.; Orosco, M.; Feng, X.; Müllen, K.; Zhou, C. J. Am. Chem. Soc. 2014, 136 (21), 7555-7558.

(33) Konnerth, R; Cervetti, C.; Narita, A.; Feng, X.; Müllen, K.; Hoyer, A.; Burghard, M.; Kern, K.; Dressel, M.; Bogani, L. Nanoscale 2015, 7 (30), 12807-12811.

(34) Osella, S.; Narita, A.; Schwab, M. G.; Hernandez, Y.; Feng, X.; Müllen, K.; Beljonne, D. ACS Nano 2012, 6 (6), 5539-5548.

(35) Ferrari, A. C.; Robertson, J. Phys. Rev. B: Condens. Matter Mater. Phys. 2000, 61 (20), 14095-14107.

(36) Basko, D. M. New J. Phys. 2009, 11 (9), 095011.

(37) Lucchese, M. M.; Stavale, F.; Ferreira, E. H.; Vilani, C.; Moutinho, M. V. O.; Capaz, R. B.; Achete, C. A.; Jorio, A. Carbon 2010, 48 (5), 1592-1597.

(38) Martins Ferreira, E. H.; Moutinho, M. V. O.; Stavale, F.; Lucchese, M. M.; Capaz, R. B.; Achete, C. A.; Jorio, A. Phys. Rev. B: Condens. Matter Mater. Phys. 2010, 82 (12), 125429.

(39) Cançado, L. G.; Jorio, A.; Ferreira, E. H. M.; Stavale, F.; Achete, C. A.; Capaz, R. B.; Moutinho, M. V. O.; Lombardo, A.; Kulmala, T. S.; Ferrari, A. C. Nano Lett. 2011, 11 (8), 3190-3196.

(40) Eckmann, A.; Felten, A.; Mishchenko, A.; Britnell, L.; Krupke, R.; Novoselov, K. S.; Casiraghi, C. Nano Lett. 2012, 12 (8), 39253930.

(41) Eckmann, A.; Felten, A.; Verzhbitskiy, I.; Davey, R.; Casiraghi, C. Phys. Rev. B: Condens. Matter Mater. Phys. 2013, 88 (3), 35426.

(42) Thomsen, C.; Reich, S. Phys. Rev. Lett. 2000, 85 (24), 52145217.

(43) Piscanec, S.; Lazzeri, M.; Mauri, F.; Ferrari, A. C.; Robertson, J. Phys. Rev. Lett. 2004, 93 (18), 185503.

(44) Ferrari, A. C.; Meyer, J. C.; Scardaci, V.; Casiraghi, C.; Lazzeri, M.; Mauri, F.; Piscanec, S.; Jiang, D.; Novoselov, K. S.; Roth, S.; Geim, A. K. Phys. Rev. Lett. 2006, 97 (18), 187401.

(45) Casiraghi, C.; Hartschuh, A.; Qian, H.; Piscanec, S.; Georgi, C.; Fasoli, A.; Novoselov, K. S.; Basko, D. M.; Ferrari, A. C. Nano Lett. 2009, 9 (4), 1433-1441.

(46) Prezzi, D.; Varsano, D.; Ruini, A.; Marini, A.; Molinari, E. Phys. Rev. B: Condens. Matter Mater. Phys. 2008, 77 (4), 1-4.

(47) Denk, R.; Hohage, M.; Zeppenfeld, P.; Cai, J.; Pignedoli, C. A.; Söde, H.; Fasel, R.; Feng, X.; Müllen, K.; Wang, S.; Prezzi, D.; Ferretti, A.; Ruini, A.; Molinari, E.; Ruffieux, P. Nat. Commun. 2014, 5, 4253.

(48) Baroni, S.; de Gironcoli, S.; Dal Corso, A.; Giannozzi, P. Rev. Mod. Phys. 2001, 73 (2), 515-562.

(49) Giannozzi, P.; Baroni, S.; Bonini, N.; Calandra, M.; Car, R.; Cavazzoni, C.; Ceresoli, D.; Chiarotti, G. L.; Cococcioni, M.; Dabo, I.; Dal Corso, A.; de Gironcoli, S.; Fabris, S.; Fratesi, G.; Gebauer, R.; Gerstmann, U.; Gougoussis, C.; Kokalj, A.; Lazzeri, M.; Martin-Samos, L.; Marzari, N.; Mauri, F.; Mazzarello, R.; Paolini, S.; Pasquarello, A.; Paulatto, L.; Sbraccia, C.; Scandolo, S.; Sclauzero, G.; Seitsonen, A. P.; Smogunov, A.; Umari, P.; Wentzcovitch, R. M. J. Phys.: Condens. Matter 2009, 21 (39), 395502.
(50) Lazzeri, M.; Mauri, F. Phys. Rev. Lett. 2003, 90 (3), 036401. 